\title{
Strukturierte Blutzuckerselbstmessung Speziell geschulte Ärzte können den Nutzen weiter erhöhen
}

- Diabetiker profitieren von einer strukturierten Blutzuckerselbstmessung (BZSM), wenn die gemessenen Werte zu einer angemessenen Verhaltens- und Therapieänderung führen. Das hatte die Studie STeP ergeben [1]. Noch effektiver könnte dies sein, wenn es den behandelnden Ärzten durch eine entsprechende Schulung und ein Entscheidungshilfesystem erleichtert würde, die ungünstige Blutzuckersituation zu erkennen und die richtigen Therapieschritte einzuleiten. Das ist ein Ergebnis der Studie Accu-Chek ${ }^{\circledast}$ DECIDE der Endokrinologin Dr. Helena W. Rodbard aus Rockville, USA, und ihren Kollegen.

In der prospektiven, kontrollierten Multicenterstudie wurden 288 Hausärzte am Computer mit 30 typischen, aber fiktiven Diabeteskasuistiken konfrontiert. In vier Gruppen eingeteilt, erhielten sie zuvor unterschiedliche Hilfsmittel zur Einschätzung der jeweiligen Stoffwechsellage. Die erste Gruppe (A) erhielt nur die visuelle Aufbereitung eines 7-Punkte-BZ-Profils über drei Tage, erfasst im Accu-Chek ${ }^{\oplus} 360^{\circ}$ View Tool von Roche. Die zweite Gruppe (B) erhielt zusätzlich die Berichte eines speziell entwickelten Entscheidungshilfesystems, das entsprechend der Werte Vorschläge zum weiteren Vorgehen machte. Die dritte Gruppe (C) erhielt stattdessen zusätzlich eine ca. 30-minütige Schulungs-
DVD. Die vierte Gruppe (D) erhielt sowohl die DVD als auch die Berichte des Entscheidungshilfesystems. Die Teilnehmer sollten in den 30 Fallbeispielen am Computer die dominierenden BZ-Abweichungen, etwa Hypoglykämie oder postprandiale Hyperglykämie, erkennen und adäquate Konsequenzen anbieten.

Von den 233 Ärzten, die das Studienprotokoll erfüllt hatten, schnitten die der Gruppe D mit allen drei Hilfsmitteln am besten ab. Signifikant mehr Ärzte (87\%) erkannten hier die primären Blutzuckerabweichungen als in den Gruppen A (51\%), B (77\%, ) und C (72\%). Beim richtigen Wählen der adäquaten Therapie lagen zwar die Ergebnisse der Gruppen B bis $\mathrm{D}$ dichter beieinander, waren aber alle signifikant besser als die der Gruppe A. Zu den Hilfsmitteln befragt gaben 90 bzw. 95\% der Teilnehmer an, von Entscheidungshilfesystem und/oder DVD zusätzlich zum $360^{\circ}$ View Tool wesentlich profitiert zu haben.

Rodbard schloss aus den Ergebnissen, dass der Fortschritt infolge einer strukturierten BZSM durch ein Training der behandelnden Ärzte in der Interpretation und Anwendung dieses Hilfsmittels noch deutlich verbessert werden könnte.

spa

Quelle: Fachpressegespräch beim EASD-Kongress, 12.9.2011 in Lissabon, Veranstalter: Roche Diabetes Care
1. Polonsky WH, Fisher L, Shikman CHet al. Diabetes Care 2011 34:262-7.

\section{GLP-1-Rezeptor-Agonist bei Typ-2-Diabetes Mit Gewichtsverlust ins Blutzuckerziel}

- Der GLP-1-Rezeptor-Agonist Lixisenatid kann bei Typ-2-Diabetikern, deren Blutzucker durch Metformin nur unzureichend eingestellt ist, den $\mathrm{HbA}_{1 c^{-}}$ Wert und das Körpergewicht signifikant senken, wie die Studie GetGoal-F1 zeigte.

In der 24-wöchigen doppelblinden kontrollierten Multicenterstudie wurden 482 Patienten mit Typ-2Diabetes zusätzlich zu Metformin mit dem einmal täglichen subkutan zu injizierenden Lixisenatid oder Placebo behandelt. Die Teilnehmer mit Verumpräparat wurden wie folgt eingestellt: Lixisenatid mit einstufiger Dosissteigerung ( $10 \mu \mathrm{g}$ für zwei Wochen, dann $20 \mu \mathrm{g}$ ) oder mit zweistufiger Steigerung (10 $\mu \mathrm{g}$ in der ersten, $15 \mu \mathrm{g}$ in der zweiten Woche, dann 20 $\mu \mathrm{g})$. Das Ergebnis: Im Vergleich zu Placebo hatte das Verumpräparat in beiden Regimen den $\mathrm{HbA}_{1 \mathrm{c}}$-Basiswert nach 24 Wochen signifikant gesenkt (einstufig: $-0,92 \%$; zweistufig: $-0,83 \%$; vs. Placebo: $-0,42 \%$; $\mathrm{p}<0,0001)$. Das $\mathrm{HbA}_{1 \mathrm{c}}-\mathrm{Ziel}$ von $\leq 6,5 \%$ bzw. $<7 \%$ erreichten beim einstufigen Therapieregime 25,6\% bzw. 47,4\% der Patienten. Beim zweistufigen Schema lag der Anteil bei 20,4\% bzw. 42,1\% versus 7,6\% bzw. $24,1 \%$ bei Placebo. Zusätzlich sank sowohl im ein- als auch im zweistufigen Therapieregime das Körpergewicht mit Lixisenatid signifikant: einstufig: $-2,63$ kg bzw. zweistufig: $-2,68 \mathrm{~kg}$ vs. Placebo: $-1,63 \mathrm{~kg}$ ( $\mathrm{p}=0,0042$ (einstufig) bzw. $\mathrm{p}=0,0025$ (zweistufig)). $5,6 \%$ der Patienten brachen die Studie im einstufigen bzw. 8,1\% im zweistufigen Regime ab (Placebo: 2,5\%). Die Häufigkeit unerwünschter Effekte lag im Bereich, der für die Wirkstoffklasse zu erwarten war und betraf v.a. Übelkeit und Erbrechen, die im Verlauf schnell verschwanden. Das Risiko einer Hypoglykämie war mit Lixisenatid nicht erhöht.

Der GetGoal-F1-Studie zufolge ist die einstufige Dosissteigerung wohl der bessere Therapieeinstieg mit Lixisenatid. Die Zulassung des Präparats mit dem voraussichtlichen Handelsnamen Lyxumia ${ }^{\oplus}$ wird für das Jahr 2012 erwartet.

fw

in Lissabon, Veranstalter: Sanofi Diabetes 